University of New Orleans

ScholarWorks@UNO

8-1995

\title{
Single-layer-coated surfaces with linearized reflectance versus angle of incidence: application to passive and active silicon rotation sensors
}

R. M.A. Azzam

University of New Orleans, razzam@uno.edu

M. M. K. Howlader

T. Y. Georgiou

Follow this and additional works at: https://scholarworks.uno.edu/ee_facpubs

Part of the Electrical and Electronics Commons, and the Optics Commons

\section{Recommended Citation}

R. M. A. Azzam, M. M. K. Howlader, and T. Y. Georgiou, "Single-layer-coated surfaces with linearized reflectance versus angle of incidence: application to passive and active silicon rotation sensors," J. Opt. Soc. Am. A 12, 1790-1798 (1995)

This Article is brought to you for free and open access by the Department of Electrical Engineering at ScholarWorks@UNO. It has been accepted for inclusion in Electrical Engineering Faculty Publications by an authorized administrator of ScholarWorks@UNO. For more information, please contact scholarworks@uno.edu. 


\title{
Single-layer-coated surfaces with linearized reflectance versus angle of incidence: application to passive and active silicon rotation sensors
}

\author{
R. M. A. Azzam, M. M. K. Howlader, ${ }^{*}$ and T. Y. Georgiou ${ }^{\dagger}$ \\ Department of Electrical Engineering, University of New Orleans, Lakefront, New Orleans, Louisiana 70148
}

Received December 9, 1994; revised manuscript received March 20, 1995; accepted March 27, 1995

\begin{abstract}
A transparent or absorbing substrate can be coated with a transparent thin film to produce a linear reflectanceversus-angle-of-incidence response over a certain range of angles. Linearization at and near normal incidence is a special case that leads to a maximally flat response for $p$-polarized, $s$-polarized, or unpolarized light. For midrange and high-range linearization with moderate and high slopes, respectively, the best results are obtained when the incident light is $s$ polarized. Application to a Si substrate that is coated with a $\mathrm{SiO}_{2}$ film leads to novel passive and active reflection rotation sensors. Experimental results and an error analysis of this rotation sensor are presented.
\end{abstract}

\section{INTRODUCTION}

In this paper it is shown that a transparent or absorbing (semiconductor or metal) substrate can be coated with a transparent thin film of the proper refractive index and thickness such that the intensity (or power) reflectance $\mathcal{R}$ for incident $p$-polarized, $s$-polarized, or unpolarized light is a linear function of the angle of incidence $\phi$ over a certain range. Such a surface with linearized angular reflectance response is the basis for a new rotation sensor. Although good results are obtained with one homogeneous film, it should be apparent that multilayer and gradedindex films offer additional flexibility that may be used, e.g., to broaden the range of linearity or to achieve a linear response at more than one wavelength.

As a basis for further discussion, we start with $\mathcal{R}$ as a function of $\phi$ for an uncoated optically isotropic substrate with complex refractive index $N_{2}=n_{2}-j k_{2}$. As an example, Fig. 1 shows $\mathcal{R}$ versus $\phi$ for light reflection in air by the plane surface of a bare $\mathrm{Si}$ substrate with $N_{2}=3.85-j 0.02$ at a $633-n m$ wavelength. ${ }^{1}$ The reflectance $\mathcal{R}_{s}$ for $s$-polarized light increases monotonically with $\phi$ between normal $(\phi=0)$ and grazing $\left(\phi=90^{\circ}\right)$ incidence, whereas $\mathcal{R}_{p}$ experiences a minimum at the pseudo-Brewster angle. ${ }^{2}$ The average reflectance $\mathcal{R}_{u}$ for incident unpolarized light, shown by the dashed curve, experiences a shallow minimum at oblique incidence.

Again, our objective is to coat the substrate with a transparent thin film of refractive index $n_{1}$ and thickness $d$ such that $\mathcal{R}$ is a linear function of $\phi$ over a certain range. It is convenient to divide the full range of $\phi$ into three ranges: an initial range I near normal incidence $\left(0 \leq \phi<30^{\circ}\right)$, a midrange II $\left(30^{\circ}<\phi<70^{\circ}\right)$, and a high range III $\left(70^{\circ}<\phi<90^{\circ}\right)$.

Linearization in range I leads to a maximally flat response (MFR) at and near normal incidence. First we note that $\mathcal{R}$ is an even function of $\phi$ :

$$
\mathcal{R}(-\phi)=\mathcal{R}(\phi)
$$

so that all the odd-numbered derivatives are zero at $\phi=0$.
(Changing the sign of $\phi$ is permitted mathematically and may be thought of physically as changing the direction of incidence from one side of the surface normal to the other in a given plane of incidence.) Therefore a Taylor-series expansion of $\mathcal{R}$ around $\phi=0$ takes the form

$\mathcal{R}(\phi)=\mathcal{R}(0)+\left(\phi^{2} / 2 !\right) \mathcal{R}^{(2)}(0)+\left(\phi^{4} / 4 !\right) \mathcal{R}^{(4)}(0)+\ldots$,

where $\mathcal{R}^{(n)}$ is the $n$th derivative of $\mathcal{R}$ with respect to $\phi$. The MFR is realized when

$$
\mathcal{R}^{(2)}(0)=\mathcal{R}^{(4)}(0)=0
$$

and is described by the Taylor series

$$
\mathcal{R}(\phi)=\mathcal{R}(0)+\left(\phi^{6} / 6 !\right) \mathcal{R}^{(6)}(0)+\ldots
$$

We further appreciate that Eq. (4) represents a truly flat response in range I by noting that the coefficient of the sixth derivative $\phi^{6} / 6 !=2.2 \times 10^{-5}$ for $\phi=0.5 \mathrm{rad}$. The MFR is illustrated by several examples in Section 2 .

Linearization in ranges II and III is based on writing a Taylor-series expansion of $\mathcal{R}(\phi)$ around a certain operating (or bias) angle of incidence $\phi_{0}$ :

$$
\begin{aligned}
\mathcal{R}(\phi)= & \mathcal{R}\left(\phi_{0}\right)+\left(\phi-\phi_{0}\right) \mathcal{R}^{(1)}\left(\phi_{0}\right)+\left[\left(\phi-\phi_{0}\right)^{2} / 2 !\right] \mathcal{R}^{(2)}\left(\phi_{0}\right) \\
& +\left[\left(\phi-\phi_{0}\right)^{3} / 3 !\right] \mathcal{R}^{(3)}\left(\phi_{0}\right)+\ldots
\end{aligned}
$$

and requiring that

$$
\mathcal{R}^{(2)}\left(\phi_{0}\right)=\mathcal{R}^{(3)}\left(\phi_{0}\right)=0 .
$$

The function of the transparent-layer coating is to force the conditions represented by Eqs. (6) [or Eqs. (3) for the MFR] to be satisfied. Specific examples are presented in Section 3.

The equations of light reflection by coated and uncoated surfaces are readily available elsewhere. ${ }^{3-5}$ All derivatives were determined analytically; the expressions are too lengthy to be listed here. 


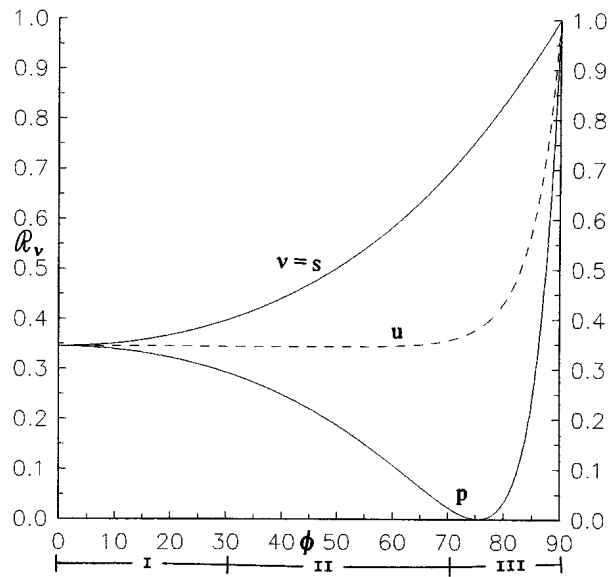

Fig. 1. Reflectance $\mathcal{R}$ for $p$-polarized, $s$-polarized, and unpolarized light as a function of the angle of incidence $\phi$ for an uncoated Si substrate with complex index of refraction $N_{2}=3.85-j 0.02$ at a 633-nm wavelength. The reflectance is linearized in any one of the ranges marked I, II, and III with a transparent-layer coating.

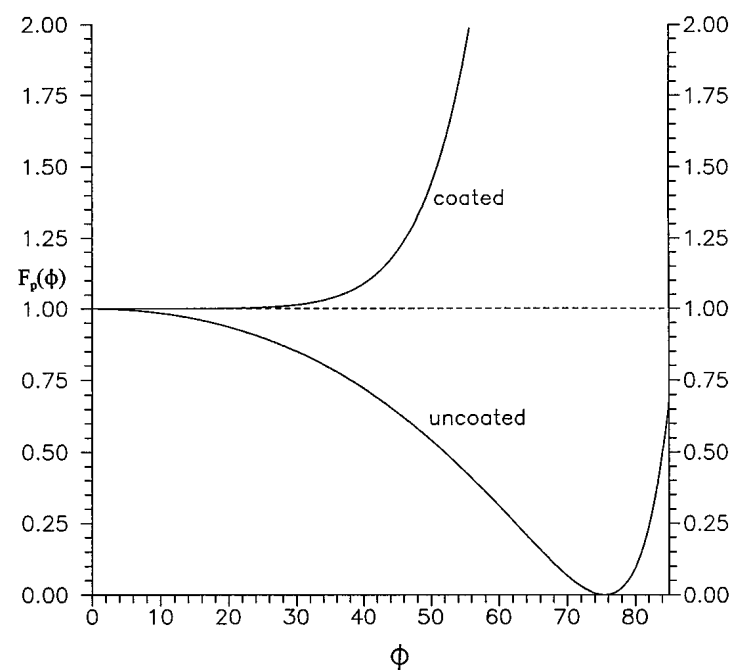

Fig. 2. Normalized response $F_{p}(\phi)$ for coated and uncoated $\mathrm{Si}$ for incident $p$-polarized light of $633-\mathrm{nm}$ wavelength. The coating, a $\mathrm{SiO}_{2}$ film of refractive index 1.460 and thickness $71.5 \mathrm{~nm}$, produces a maximally flat reflectance in range I $\left(0<\phi<30^{\circ}\right)$.

\section{LINEARIZATION AT AND NEAR NORMAL INCIDENCE (RANGE I): MAXIMALLY FLAT RESPONSE}

It is convenient to work with the normalized response function

$$
F_{\nu}(\phi)=\mathcal{R}_{\nu}(\phi) / \mathcal{R}_{\nu}(0),
$$

where $\nu=p, s, u$ represent $p$-polarized, $s$-polarized, and unpolarized light, respectively. For a Si substrate and $p$-polarized incident light of 633-nm wavelength, Eqs. (3) are satisfied with a film of refractive index $n_{1}=1.460$ (fortuitously that of $\mathrm{SiO}_{2}$ ) and thickness $d=71.49 \mathrm{~nm}$. The resulting MFR in range I and beyond is shown in Fig. 2, along with that of a bare $\mathrm{Si}$ substrate; in this case $\mathcal{R}(0)=16.85 \%$

For the $s$ polarization the MFR is achieved with a film of refractive index $n_{1}=1.858$ (which corresponds to sili- con nitride or oxynitride ${ }^{6}$ ) and thickness $d=123.68 \mathrm{~nm}$. The functions $F_{s}(\phi)$ are shown in Fig. 3 for the coated and uncoated $\mathrm{Si}$ substrates. It is apparent that an excellent MFR is attained in range I $\left(0<\phi<30^{\circ}\right)$ with a single-layer coating; the constant reflectance level $\mathcal{R}_{s}(\phi) \approx \mathcal{R}(0)=18.54 \%$.

For incident unpolarized light, Eqs. (3) are satisfied with a film with $n_{1}=3.795$ (which differs slightly from that of $\mathrm{Si}$ ) and $d=37.11 \mathrm{~nm}$. This coating, which is not practical, provides only a marginal improvement (disappearance of the shallow minimum in Fig. 1). In this case $\mathcal{R}(0)=33.39 \%$, which differs slightly from that of bare Si. That uncoated Si provides a nearly MFR for incident unpolarized light is consistent with previously published work. ${ }^{7}$

The same procedure has been applied to other substrates. For example, Fig. 4 shows $F_{s}$ for an $\mathrm{Al}$ substrate $\left(N_{2}=1.5-j 7.6\right.$ at a 633 -nm wavelength $\left.{ }^{8}\right)$ with and without a single-layer coating $\left(n_{1}=2.138, d=96.20 \mathrm{~nm}\right)$ that achieves a MFR at and near normal incidence. $\mathcal{R}_{s}(\phi) \approx$ $\mathcal{R}_{s}(0)=84.95 \%$ for the coated surface for $0<\phi<30^{\circ}$.

Surfaces with a MFR can be useful as angle-insensitive reflectance standards and in scanning applications that may require beam deflection without a change of the reflected light intensity.

\section{LINEARIZATION IN THE MIDRANGE AND HIGH RANGE OF INCIDENCE ANGLES}

Coatings that achieve linearity in ranges II and III by satisfying Eqs. (6) have been found for different substrates including $\mathrm{Si}, \mathrm{Al}, \mathrm{ZnS}$, and $\mathrm{SiO}_{2}$ (glass) at $633 \mathrm{~nm}$ and at other (laser) wavelengths. For all the cases considered, solutions are readily obtained for incident $s$-polarized light. However, no solutions could be found for the $p$ polarization, and only occasional solutions are found for unpolarized light.

Equations (6) [or Eqs. (3)] are simultaneous nonlinear equations that are solved by numerical iteration. In the

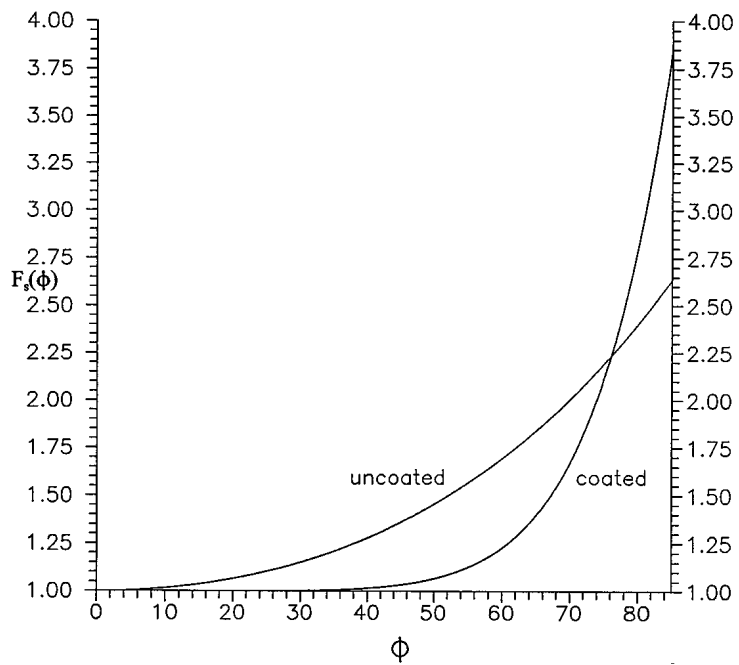

Fig. 3. Normalized response $F_{s}(\phi)$ for coated and uncoated $\mathrm{Si}$ for incident $s$-polarized light of $633-\mathrm{nm}$ wavelength. The coating, an oxynitride film of refractive index 1.858 and thickness $123.7 \mathrm{~nm}$, produces a maximally flat reflectance in range I $\left(0<\phi<30^{\circ}\right)$. 


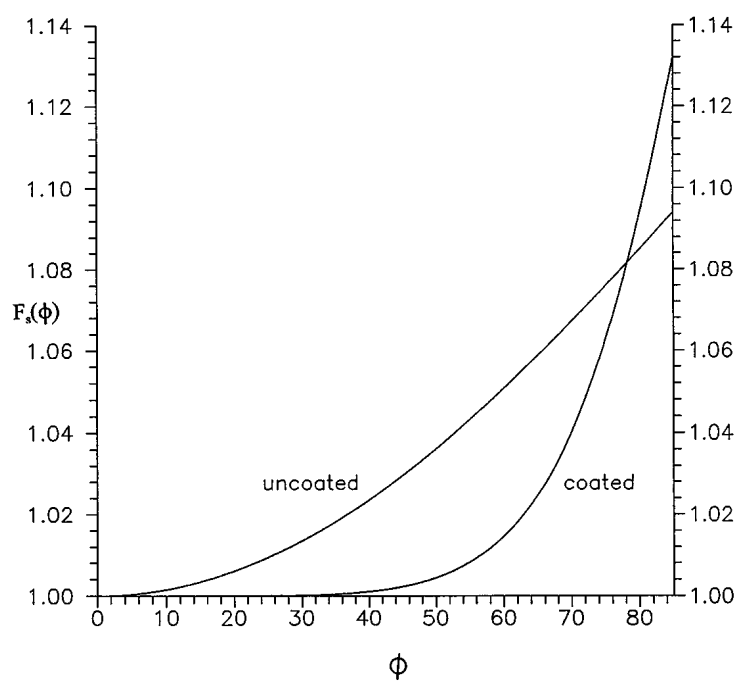

Fig. 4. Normalized response $F_{s}(\phi)$ for coated and uncoated $\mathrm{Al}$ for incident $s$-polarized light of $633-\mathrm{nm}$ wavelength. The coating, a transparent film of refractive index 2.138 and thickness $96.2 \mathrm{~nm}$, produces a maximally flat reflectance in range I $\left(0<\phi<30^{\circ}\right)$.

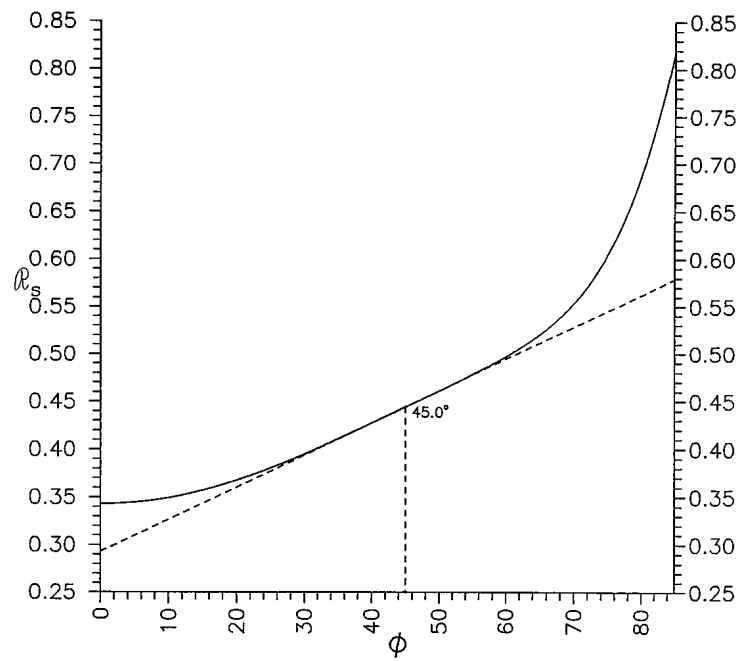

Fig. 5. Linearization of the reflectance $\mathcal{R}_{s}$ of $\mathrm{Si}$ for incident $s$-polarized light of $633-\mathrm{nm}$ wavelength around the angle $\phi_{0}=45^{\circ}$ with a dense $\mathrm{MgF}_{2}$ film of refractive index 1.394 and thickness $235.2 \mathrm{~nm}$.

present case, one can assign a value to $\phi_{0}$ and solve Eqs. (6) for the film's refractive index $n_{1}$ and thickness $d$. Alternatively, one can assign a value to $n_{1}$ and solve Eqs. (6) for $\phi_{0}$ and $d$. The latter approach is important because it is easier to adjust the angle of incidence than it is to adjust the film's refractive index.

As an example of linearization in range II we consider a Si substrate, incident $s$-polarized light of 633 -nm wavelength, and the convenient operating angle of incidence $\phi_{0}=45^{\circ}$. Solving Eqs. (6) for the parameters of the transparent film gives $n_{1}=1.394$ (which corresponds to dense $\left.\mathrm{MgF}_{2}\right)^{9}$ and $d=235.2 \mathrm{~nm}$. Figure 5 shows the resulting linearized $\mathcal{R}_{s}$-versus- $\phi$ curve around $\phi_{0}=45^{\circ}$ at which $\mathcal{R}_{s}=44.43 \%$ and the slope of the linear response is $0.366 \% \mathrm{deg}^{-1}$. Figures 6(a), 6(b), and 6(c), respectively, show the first, second, and third angle-of-incidence derivatives $\mathcal{R}_{s}{ }^{\prime}, \mathcal{R}_{s}{ }^{\prime \prime}$, and $\mathcal{R}_{s}{ }^{\prime \prime \prime}$ as functions of $\phi$ for this coated-
Si surface. Notice the plateau of constant slope $\mathcal{R}_{s}^{\prime}$ in Fig. 6(a) and the zeros of $\mathcal{R}_{s}^{\prime \prime}$ and $\mathcal{R}_{s}^{\prime \prime \prime}$ at $\phi_{0}=45^{\circ}$ in Figs. 6(b) and 6(c), as required by design.

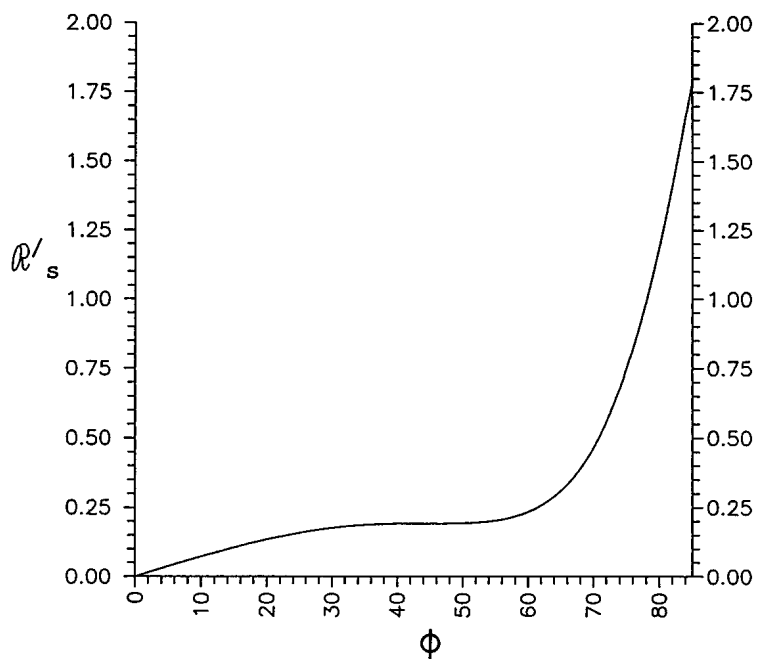

(a)

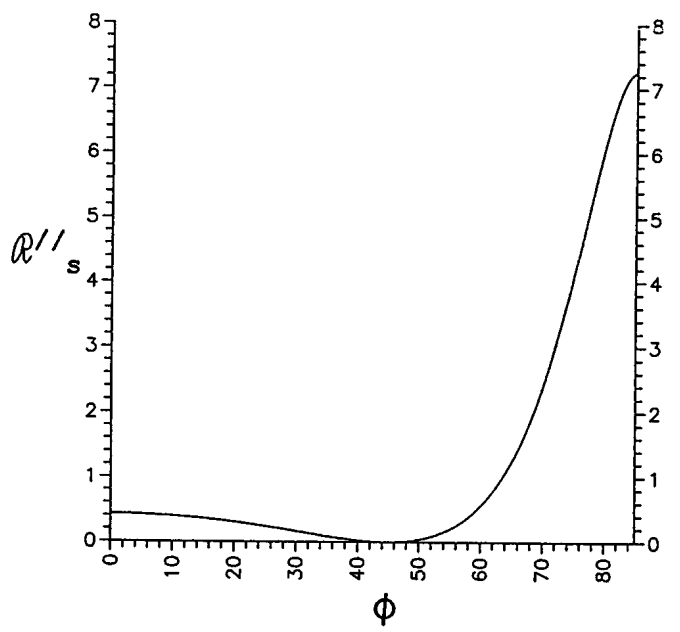

(b)

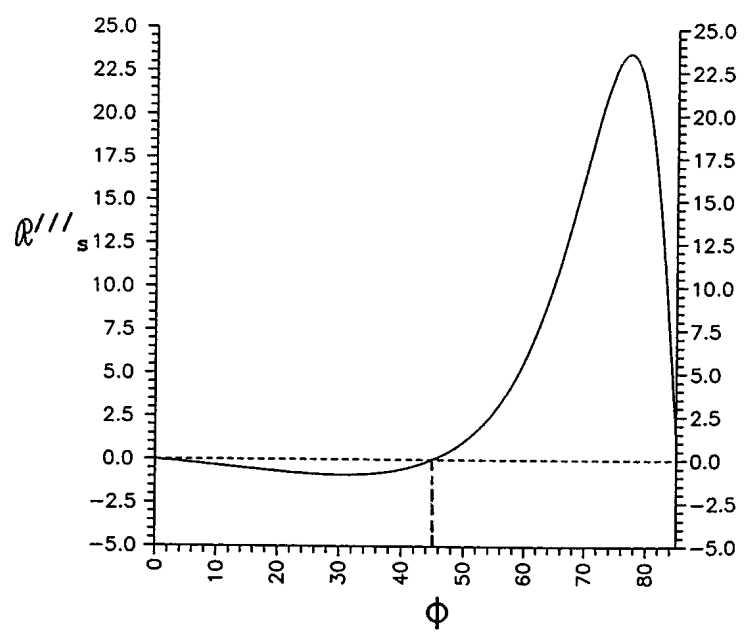

(c)

Fig. 6. (a) First derivative $\mathcal{R}_{s}{ }^{\prime}$, (b) second derivative $\mathcal{R}_{s}{ }^{\prime \prime}$, and (c) third derivative $\mathcal{R}_{s}{ }^{\prime \prime \prime}$ as functions of angle of incidence $\phi$ for the linearized reflectance response shown in Fig. 5. 


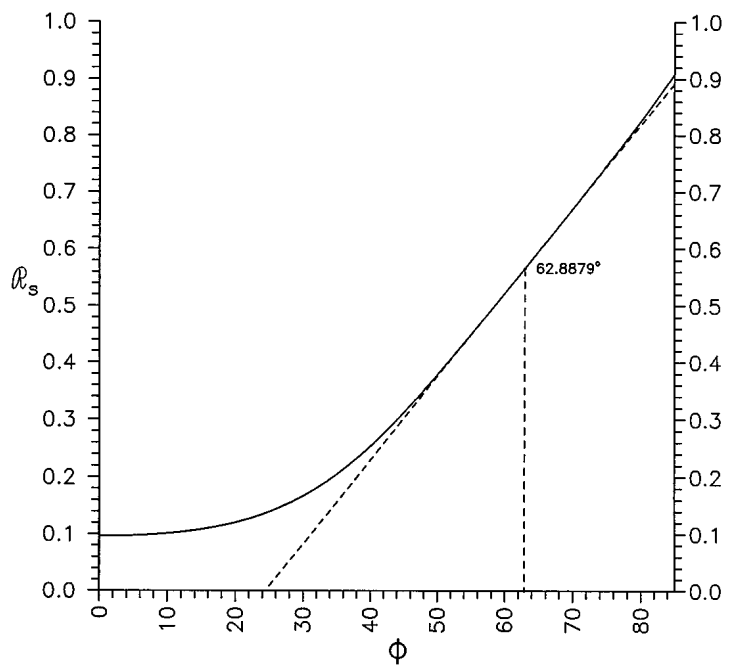

Fig. 7. Linearization of the reflectance $\mathcal{R}_{s}$ of $\mathrm{Si}$ for incident $s$-polarized light of 633-nm wavelength around the angle $\phi_{0}=$ $62.89^{\circ}$ with a $\mathrm{SiO}_{2}$ film of refractive index 1.46 and thickness $311.4 \mathrm{~nm}$.

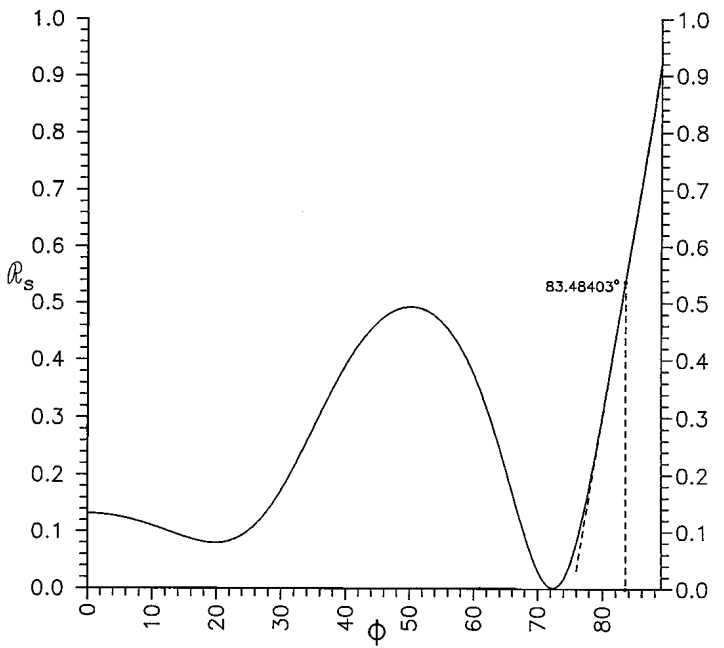

Fig. 8. Linearization of the reflectance $\mathcal{R}_{s}$ of $\mathrm{Si}$ for incident $s$-polarized light of 633-nm wavelength around the angle $\phi_{0}=$ $83.48^{\circ}$ with $\mathrm{a} \mathrm{SiO}_{2}$ film of refractive index 1.46 and thickness $1002.0 \mathrm{~nm}$.

Another important example is that of a $\mathrm{SiO}_{2}$ film (with refractive index 1.46) upon a $\mathrm{Si}$ substrate at the same 633-nm wavelength. In this case Eqs. (6) are solved for the operating angle $\phi_{0}=62.888^{\circ}$ and the film thickness $d=311.4 \mathrm{~nm}$. The corresponding linearized $\mathcal{R}_{s}(\phi)$ response, which appears in Fig. 7, has a higher slope of $1.47 \% \mathrm{deg}^{-1}$ at $\phi_{0}$. The linear range (LR) is defined as that range of $\phi$ over which the actual response deviates from its linear approximation (the tangent to the curve at $\phi_{0}$ ) by no more than $1 \%$. For the present example the $\mathrm{LR}$ is given by $55.7^{\circ}<\phi<70.3^{\circ}$. This particular $\mathrm{SiO}_{2}-\mathrm{Si}$ design is the subject of an error analysis given in Section 4. It is also the design that we adopt to implement an optical rotation sensor for measuring the angular speed and acceleration of a rotating shaft as described in Section 5 .

To obtain a much higher slope (at the expense of a reduction in the LR) one has to linearize in range III. An example of this uses the same $\mathrm{SiO}_{2}-\mathrm{Si}$ film-substrate system but with a higher oxide thickness $d=1002.0 \mathrm{~nm}$ and a higher operating angle $\phi_{0}=83.484^{\circ}$ at the same $633-\mathrm{nm}$ wavelength. The reflectance curve $\mathcal{R}_{s}(\phi)$ appears in Fig. 8. The linear response occurs at high angles (LR $81.1^{\circ}<\phi<85.6^{\circ}$ ) with a slope of $6.75 \% \mathrm{deg}^{-1}$. It is interesting to note that $\mathcal{R}_{s} \approx 0$ at $72^{\circ}$, which indicates that this $\mathrm{SiO}_{2}-\mathrm{Si}$ system acts as an accidental polarizer.

For ease of reference, the results cited above are summarized in Table 1. This table also summarizes other results that we obtained for linearization of the $\mathcal{R}_{s}$-versus- $\phi$ curve by using $\mathrm{Al}, \mathrm{ZnS}$, and $\mathrm{SiO}_{2}$ (glass) substrates at a 633-nm wavelength.

For the glass substrate $\left(N_{2}=1.5\right)$ the low refractive index of the film, $n_{1}=1.127$, does not correspond to that of any known thin solid film but can be realized by creation of a two-dimensional subwavelength-structured surface on glass, as is now well known in diffractive optics. ${ }^{10}$

Of the results included in Table 1 the broadest LR $\left(48.9^{\circ}<\phi<81.3^{\circ}\right)$ is obtained for an $\mathrm{Al}$ substrate that is coated with a transparent film of refractive index 2.20 [e.g., low-density $\mathrm{ZnS}$ (Ref. 9)] and of 160.1-nm thickness. The resulting linearized response $R_{s}(\phi)$ is shown in Fig. 9; it has a slope of $0.16 \% \mathrm{deg}^{-1}$ at $\phi_{0}=62.385^{\circ}$. This slope is small because of the high reflectance of $\mathrm{Al}$ at normal incidence.

Finally, we show an example of the linearization of reflectance for incident unpolarized light $\mathcal{R}_{u}$ (Fig. 10), using a Si substrate at 633 -nm wavelength. With $\phi_{0}=$ $65^{\circ}$, the required transparent coating has a refractive index $n_{1}=1.381\left(\mathrm{MgF}_{2}\right)$ and a thickness $d=384.23 \mathrm{~nm}$. In this case $\mathcal{R}_{u}\left(\phi_{0}\right)=30.27 \%$ and the slope $\mathcal{R}_{u}{ }^{\prime}\left(\phi_{0}\right)=$ $1.11 \% \mathrm{deg}^{-1}$.

Table 1. Coatings for Linearized-Reflectance-versus-Angle Response for Incident s-Polarized Light ${ }^{a}$

\begin{tabular}{clllrrrr}
\hline Substrate & \multicolumn{1}{c}{$N_{s}$} & \multicolumn{1}{c}{$n_{1}$} & $\phi_{0}(\mathrm{deg})$ & $d(\mathrm{~nm})$ & $\mathcal{R}_{s 0}(\%)$ & $\mathcal{R}_{s 0^{\prime}}(\%)$ & $\mathrm{LR}( \pm 1 \%)$ \\
\hline $\mathrm{Si}$ & $3.85-j 0.02$ & 1.394 & 45.000 & 235.17 & 44.43 & 0.19267 \\
$\mathrm{Si}$ & $3.85-j 0.02$ & 1.480 & 65.000 & 312.79 & 57.52 & 0.90922 \\
$\mathrm{Si}$ & $3.85-j 0.02$ & 1.46 & 62.888 & 311.36 & 56.60 & 0.84255 & $57.25-50.91$ \\
$\mathrm{Si}$ & $3.85-j 0.02$ & 1.46 & 83.484 & 1002.00 & 53.74 & 3.86667 & $81.70-70.30$ \\
$\mathrm{Al}$ & $1.5-j 7.6$ & 2.20 & 62.385 & 160.14 & 95.43 & 0.09417 & $48.90-81.25$ \\
$\mathrm{Al}$ & $1.5-j 7.6$ & 1.503 & 45.000 & 219.07 & 93.39 & 0.05688 \\
$\mathrm{ZnS}$ & 2.35 & 1.46 & 73.699 & 638.50 & 47.66 & 1.70587 & $69.53-54.35$ \\
$\mathrm{SiO}_{2}$ & 1.5 & 1.127 & 64.970 & 464.05 & 22.70 & 0.63914 \\
\hline
\end{tabular}

${ }^{a}$ The wavelength of incident monochromatic light is taken to be $633 \mathrm{~nm}, N_{s}$ is the refractive index of the substrate, $n_{1}$ is that of the film, $\phi_{0}$ is the angle of incidence for which the second and third derivatives are zero, $d$ is the film thickness, $\mathcal{R}_{s 0}$ is the intensity reflectance at the design angle, $\mathcal{R}_{s 0}{ }^{\prime}$ is first derivative of reflectance at $\phi_{0}$, and LR is the linear range in degrees. 


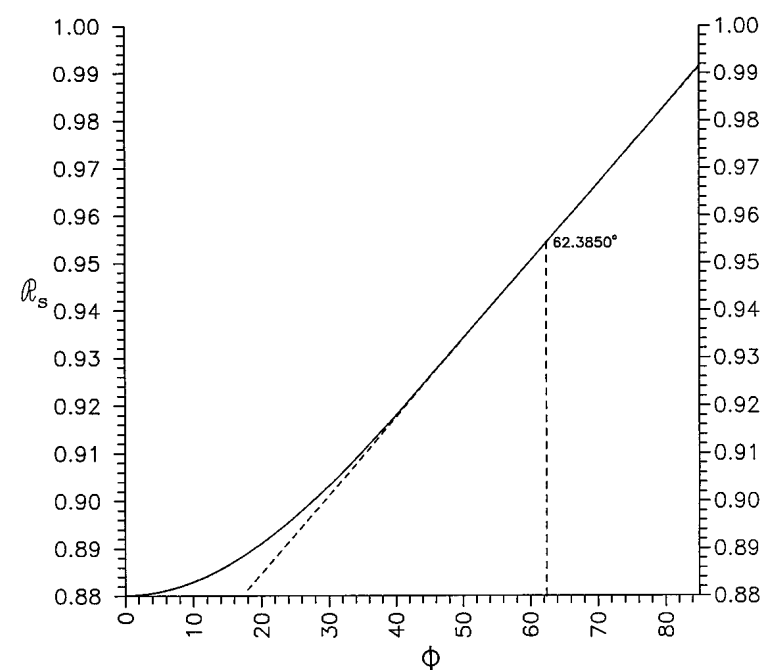

Fig. 9. Linearization of the reflectance $\mathcal{R}_{s}$ of $\mathrm{Al}$ for incident $s$-polarized light of $633-\mathrm{nm}$ wavelength around the angle $\phi_{0}=62.38^{\circ}$ with a low-density $\mathrm{ZnS}$ film of refractive index 2.20 and thickness $160.1 \mathrm{~nm}$. Notice the broad linear range (with a modest slope of $0.16 \% \mathrm{deg}^{-1}$ ).

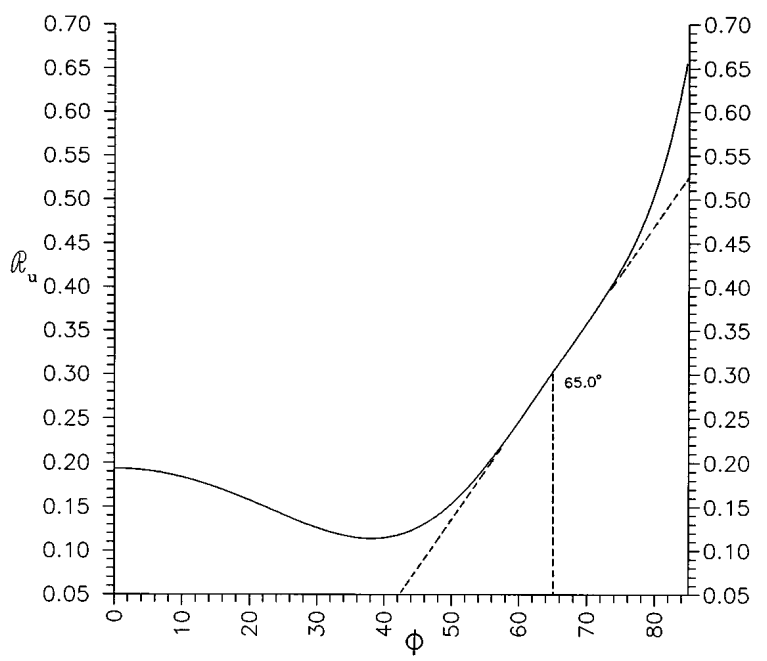

Fig. 10. Linearization of the reflectance $\mathcal{R}_{u}$ of Si for incident unpolarized light of 633-nm wavelength around the angle $\phi_{0}=65^{\circ}$ with a $\mathrm{MgF}_{2}$ film of refractive index 1.381 and thickness $384.2 \mathrm{~nm}$.

\section{ERROR ANALYSIS FOR A $\mathrm{SiO}_{2}-\mathrm{Si}$ SURFACE WITH LINEARIZED REFLECTANCE}

Now we consider the effect of introducing small errors $( \pm 5 \%)$ of the film's refractive index or thickness on the linearized $\mathcal{R}_{s}(\phi)$ response. The starting design is that which uses a 311.4-nm $\mathrm{SiO}_{2}$ film upon $\mathrm{Si}$ at a $633-\mathrm{nm}$ wavelength. Figure 11 shows the shifted response caused by refractive-index errors, and Fig. 12 shows those caused by film-thickness errors. In both cases the middle curve represents the design curve in the absence of errors. It is evident that the film's refractive-index and thickness errors change the slope and LR but that reasonably good linearity is retained.

Figure 13 shows the effect of changing the direction of linear polarization of incident light by $\pm 5^{\circ}$ from that of the normal to the plane of incidence (i.e., the pure $s$ state).
Fortunately, the effect on the linearized $\mathcal{R}_{s}(\phi)$ response is negligible.

\section{REFLECTION ROTATION SENSOR BASED ON A $\mathrm{SiO}_{2}-\mathrm{Si}$ SURFACE WITH LINEARIZED REFLECTANCE FOR INCIDENT $s$-POLARIZED LIGHT}

The principle of operation of this sensor is described with reference to Fig. 14. Sensor element $S$ with linearized reflectance is mounted upon a rotating shaft such that the (vertical) axis of rotation is in the plane of the sensor surface. Incident light from a $\mathrm{He}-\mathrm{Ne}$ laser (633-nm wavelength) is (vertically) polarized perpendicular to the (horizontal) plane of incidence. Lens $\mathrm{L}$ is placed in the reflected beam to image the point of reflection onto a linear photodetector D (a silicon photodiode). The 633-nm narrow-band spectral filter $\mathrm{F}$ permits measurements to be done in ambient light. The revolving reflected beam is intercepted by the lens and is focused onto the detector only over a finite range of angles $\phi_{0} \pm \beta$, where $\phi_{0} \approx 63^{\circ}$

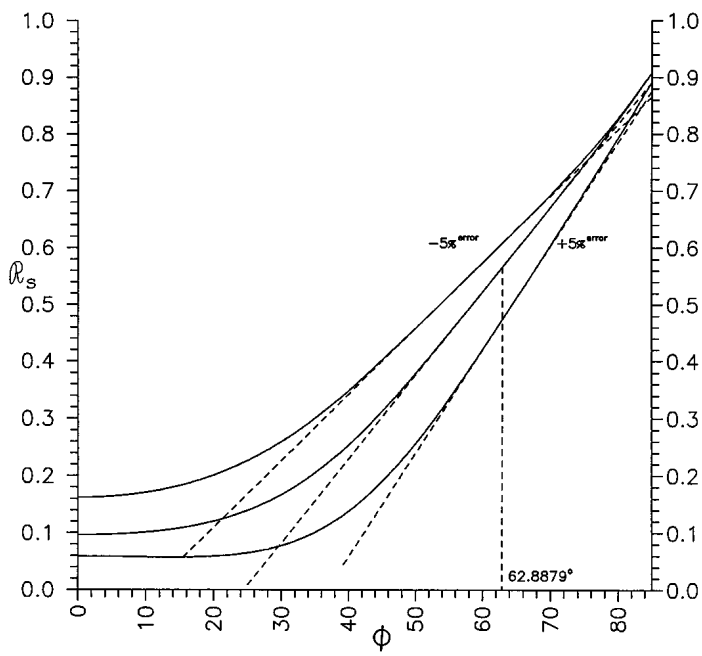

Fig. 11. Effect of $\pm 5 \%$ film refractive-index error on the linearized $s$-reflectance response of a $\mathrm{Si}$ substrate coated with a $\mathrm{SiO}_{2}$ film of thickness $311.4 \mathrm{~nm}$ at a $633-\mathrm{nm}$ wavelength.

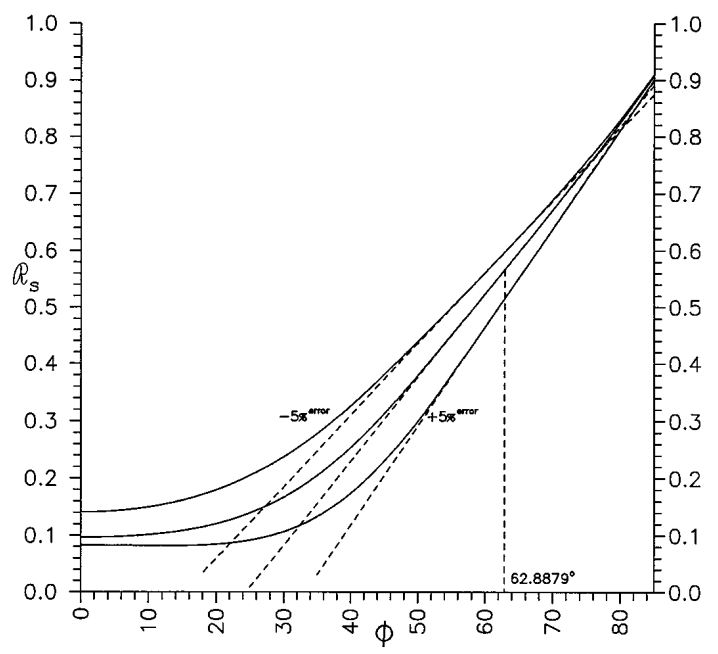

Fig. 12. Effect of $\pm 5 \%$ film-thickness error on the linearized $s$-reflectance response of a $\mathrm{Si}$ substrate coated with a $\mathrm{SiO}_{2}$ film of thickness $311.4 \mathrm{~nm}$ at a $633-\mathrm{nm}$ wavelength. 


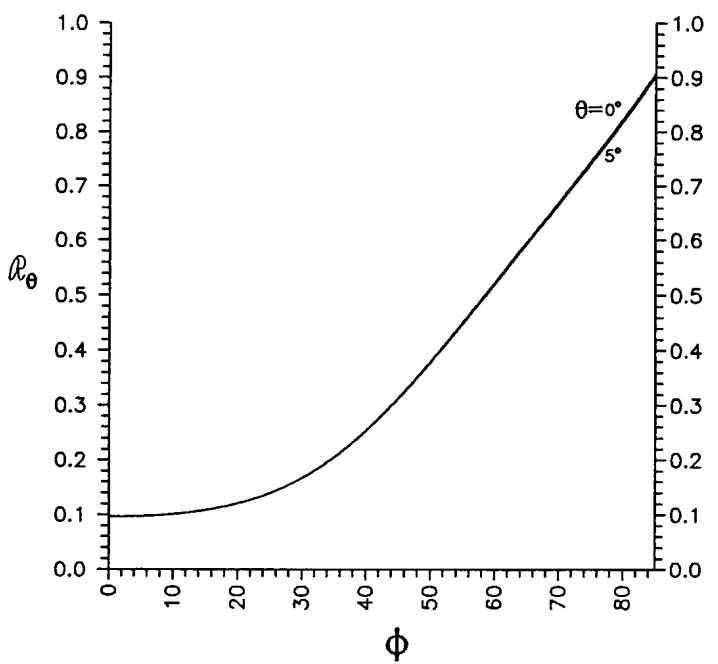

Fig. 13. Effect of $\pm 5^{\circ}$ shift $\theta$ of the orientation of the incident electric field vector from the $s$ direction on the linearized reflectance response of a $\mathrm{Si}$ substrate coated with a $\mathrm{SiO}_{2}$ film of thickness $311.4 \mathrm{~nm}$ at a $633-\mathrm{nm}$ wavelength.

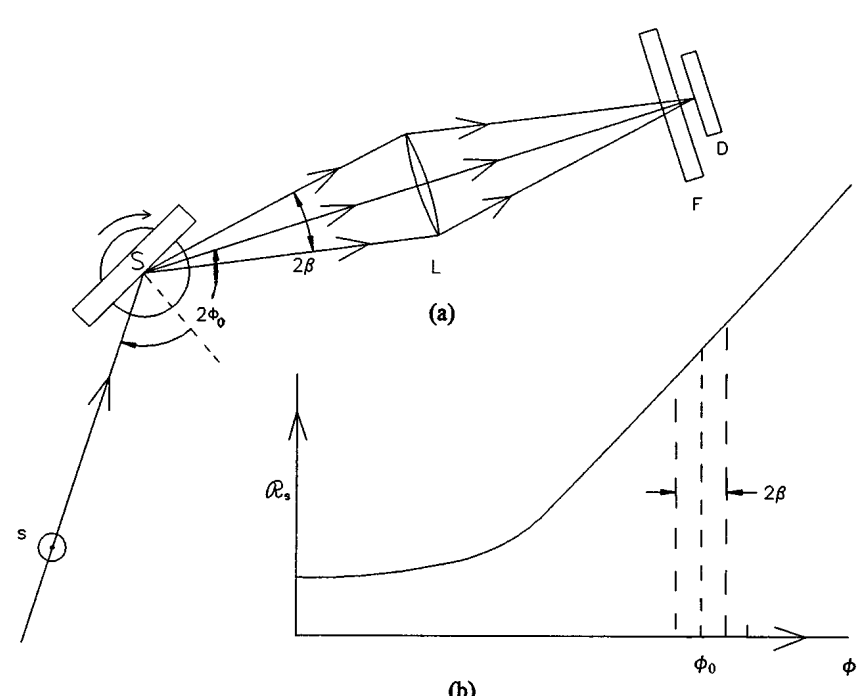

Fig. 14. (a) Experimental setup for measuring the angular speed and acceleration of a rotating shaft by a reflective sensor with linearized reflectance for incident $s$-polarized light. $\mathrm{L}$, focusing lens; F, narrow-band filter to isolate the $633-\mathrm{nm}$ reflected radiation; D, linear photodiode. (b) The linear reflectance response.

and $\beta \approx 7^{\circ}$. In our experiment we used a Si surface with a $311-\mathrm{nm} \mathrm{SiO}_{2}$ coating, which has a linear response $\mathcal{R}_{s}(\phi)$ over the indicated range of angles.

The detected signal as a function of time $t$ is given by

$$
i(t)=C I_{0 s} \mathcal{R}_{s},
$$

where $I_{0 s}$ is the intensity (or power) of incident $s$-polarized light (assumed to be constant) and $C$ is a constant that is characteristic of the detector and also accounts for the insertion loss that is due to lens $\mathrm{L}$ and filter $\mathrm{F}$. The rate of change of the signal is given by

$$
\begin{aligned}
\mathrm{d} i / \mathrm{d} t & =C I_{0 s}\left(\mathrm{~d} \mathcal{R}_{s} / \mathrm{d} \phi\right)(\mathrm{d} \phi / \mathrm{d} t), \\
& =\omega C I_{0 s} \mathcal{R}_{s}{ }^{\prime}\left(\phi_{0}\right),
\end{aligned}
$$

where $\omega=\mathrm{d} \phi / \mathrm{d} t$ is the angular velocity to be measured. If we define

$$
i_{0}=C I_{0 s} \mathcal{R}_{s}\left(\phi_{0}\right),
$$

then, by division, Eqs. (10) and (11) lead to the following solution for $\omega$ :

$$
\omega=\left[(\mathrm{d} i / \mathrm{d} t) / i_{0}\right] /\left[\mathcal{R}_{s}{ }^{\prime}\left(\phi_{0}\right) / \mathcal{R}_{s}\left(\phi_{0}\right)\right] .
$$

Equation (12) provides the basis for measuring the angular speed $\omega$ by use of the reflection sensor system of Fig. 14. The numerator of the right-hand side is determined by analysis of the electrical output pulse of the photodetector for each rotation of the shaft, and the denominator is a characteristic of the reflective sensor element.

Because the system measures $\omega$ once for each full rotation of the shaft, the angular acceleration can be readily calculated from data obtained from two successive pulses from the simple relation

$$
\alpha=\mathrm{d} \omega / \mathrm{d} t \approx\left(\omega_{2}-\omega_{1}\right) / \Delta t,
$$

where $\Delta t$ is the time separation of the two consecutive pulses.

We have implemented the technique presented above to measure the rotational speed of a small variable-speed motor. A typical output pulse is shown in Fig. 15, which leads to a measured speed of $840 \mathrm{rpm}$. Measurements at different speeds have been carried out, and the results obtained by our sensor were compared with those obtained by a commercial tachometer, ${ }^{11}$ as shown in Fig. 16 . The good agreement (to within $\pm 4 \%$ ) confirms the validity of this simple reflection sensor.

If the passive $\mathrm{SiO}_{2}-\mathrm{Si}$ reflector is replaced with an active $\mathrm{Si}$ photodiode (which is coated with an oxide layer of the same 311-nm thickness) one can measure the angular speed associated with the torsional oscillation of a shaft or a column without the use of any of the reflection optics that appear in Fig. 14. In this case the output signal of

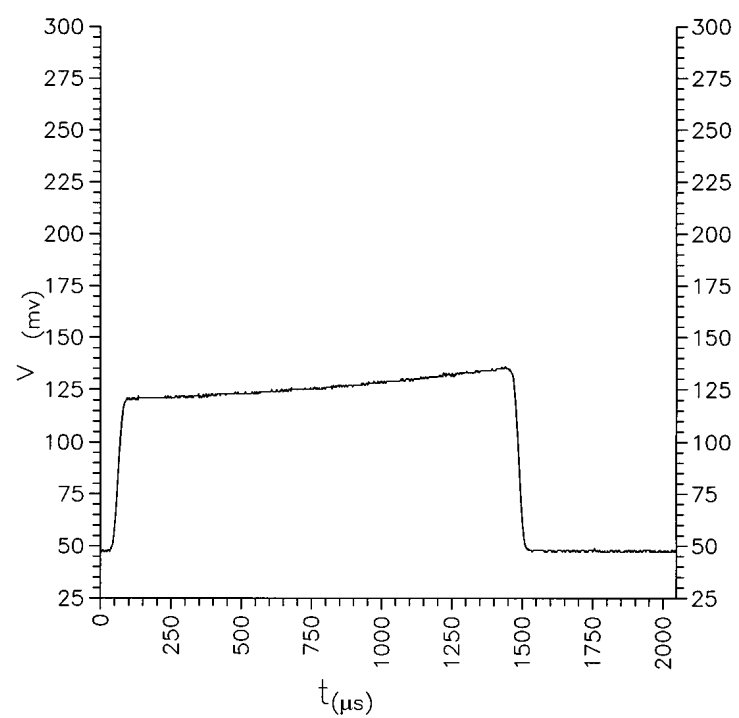

Fig. 15. Typical output electrical pulse of photodiode D in the experiment described in Fig. 14 obtained at a rotational speed of $840 \mathrm{rpm}$. 


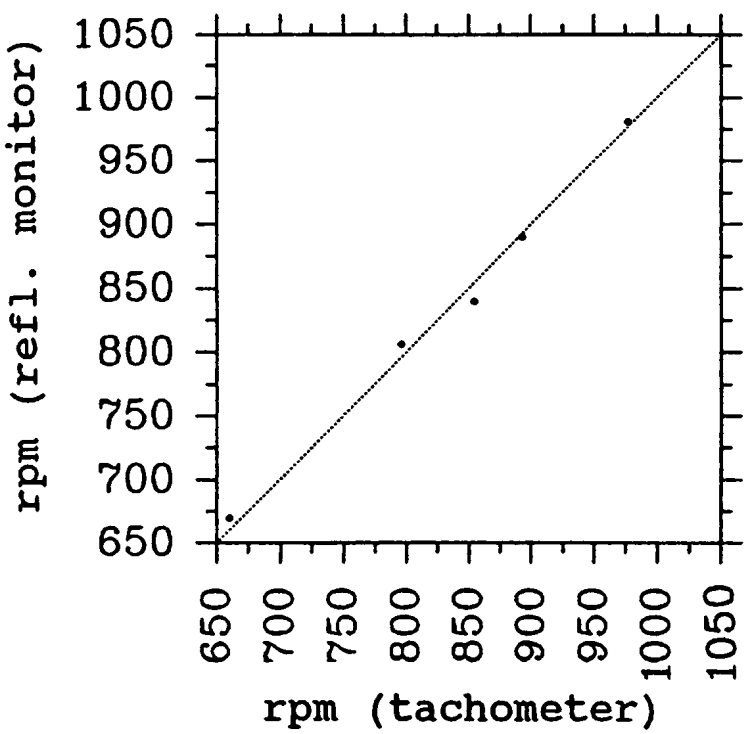

Fig. 16. Correlation of the results obtained with the rotation sensor system of Fig. 14 with those obtained with a commercial tachometer.

the shaft-mounted reflective $\mathrm{Si}$ photodiode becomes proportional to $\left(1-\mathcal{R}_{s}\right)$ instead of to $\mathcal{R}_{s}$, and the analysis of Eqs. (8)-(12) can be modified accordingly. Because of wiring constraints, continuous (unlimited) rotation of the shaft is not permitted. To operate the active sensor in ambient light we can interrupt the input signal beam by a chopper, and we can measure the output signal of the shaft-mounted detector at the modulation frequency by using lock-in signal detection.

\section{CONCLUSIONS}

We have shown that the reflectance of a surface as a function of incidence angle can be tailored to be linear over a certain range by a transparent thin-film coating of the appropriate refractive index and thickness. Linearization at and near normal incidence is possible for any incident polarization and results in a maximally flat response. The more practically important linearization in the middle and high ranges of incidence angle is achieved for $s$-polarized light. Results are presented for coatings upon $\mathrm{Si}$ and other substrates at the $\mathrm{He}-\mathrm{Ne}$ laser wave- length of $633 \mathrm{~nm}$. A particular design that uses a $\mathrm{SiO}_{2}$ film of 311-nm thickness upon Si to give a linear response over the range $63 \pm 7^{\circ}$ is used as a sensor element to measure the angular speed of a rotating shaft. An error analysis indicates that small errors of the film thickness, film refractive index, or incident light polarization can be tolerated.

\section{ACKNOWLEDGMENT}

This paper was presented at the Annual Meeting of the Optical Society of America in Dallas, Texas, October 2-7, 1994.

*Present address, Center for Research in Electro-Optics and Lasers, University of Central Florida, Orlando, Florida 32826.

${ }^{\dagger}$ Present address, Measurementation, Inc., Chino, California 91708-0031.

\section{REFERENCES}

1. G. E. Jellison, Jr., "Optical functions of silicon determined by two-channel polarization modulation ellipsometry," Opt. Mater. 1, 41-47 (1992).

2. H. B. Holl, "Specular reflection and characteristics of reflected light," J. Opt. Soc. Am. 57, 683-690 (1967); J. M. Bennett and H. E. Bennett, "Polarization," in Handbook of Optics, W. G. Driscoll and W. Vaughan, eds. (McGraw-Hill, New York, 1978).

3. M. Born and E. Wolf, Principles of Optics (Pergamon, New York, 1975), Chap. 1, pp. 36-70.

4. R. M. A. Azzam and N. M. Bashara, Ellipsometry and Polarized Light (North-Holland, Amsterdam, 1987), Chap. 4, pp. 269-288.

5. J. Lekner, Theory of Reflection (Nijhoff, Dordrecht, The Netherlands, 1987), Chap. 2, pp. 44-50.

6. D. E. Bossi, J. M. Hammer, and J. M. Shaw, "Optical properties of silicon oxynitride dielectric waveguides," Appl. Opt. 26, 609-611 (1987)

7. R. M. A. Azzam, "Limaçon of Pascal locus of the complex refractive indices of interfaces with maximally flat reflectanceversus-angle curves for incident unpolarized light,” J. Opt. Soc. Am. A 9, 957-963 (1992).

8. E. Palik, ed., Handbook of Optical Constants of Solids (Academic, New York, 1985), p. 398.

9. E. Ritter, "Dielectric film materials for optical applications," in Physics of Thin Films, G. Hass and M. H. Francombe, eds. (Academic, New York, 1975), Vol. 8, pp. 1-49.

10. See, for example, the feature on diffractive optics: design, fabrication, and applications, Appl. Opt. 32(14) 1993.

11. M25 Encoder, Madison Electric, Madison, Ohio 44057. 